Cahiers de recherches médiévales

\title{
La Table Ronde dans Les Merveilles de Rigomer
}

\section{Christine Ferlampin-Acher}

\section{OpenEdition}

Journals

Édition électronique

URL : https://journals.openedition.org/crm/2652

DOI : $10.4000 / \mathrm{crm} .2652$

ISSN : 1955-2424

Éditeur

Honoré Champion

Édition imprimée

Date de publication : 15 décembre 2007

Pagination : 49-59

ISSN : 1272-9752

Référence électronique

Christine Ferlampin-Acher, "La Table Ronde dans Les Merveilles de Rigomer », Cahiers de recherches médiévales [En ligne], 14 | 2007, mis en ligne le 15 décembre 2010, consulté le 15 décembre 2022. URL : http://journals.openedition.org/crm/2652 ; DOI : https://doi.org/10.4000/crm.2652 


\section{酷M}

\section{La Table Ronde dans Les Merveilles de Rigomer}

La Table Ronde, apparue en littérature avec Wace ${ }^{1}$ et présente à trois reprises chez Chrétien de Troyes ${ }^{2}$, a connu un vif succès, au point de devenir un véritable mythe. Les recherches à son sujet sont diverses: outre les nombreux travaux consacrés à son origine et à son évolution, entre mondes celtique (en particulier avec les travaux de R. S. Loomis et J. Marx ${ }^{3}$ ) et chrétien ${ }^{4}$, elle a retenu l'attention des historiens et des archéologues ${ }^{5}$, tant il est vrai que l'utilisation politique du mythe est riche. Par ailleurs le succès de ce motif, toujours vivant, certainement du fait de Malory, suscite de nombreuses études centrées sur le cinéma ou sur les œuvres littéraires postérieures au Moyen Âge.

Pourtant la Table Ronde est d'abord un motif littéraire dont la plasticité assure la permanence dans les romans français du XII ${ }^{\mathrm{e}}$ au $\mathrm{XV}^{\mathrm{e}}$ siècle $^{6}$ et elle paraît liée à la constitution et à l'évolution du roman comme genre : avec Wace, elle est dès l'origine rapprochée des fables bretonnes, et donc à la fiction; sa circularité et la rime «ronde/ monde» qui lui est souvent associée en font un symbole fécond de

\footnotetext{
${ }^{1}$ Wace, Brut, éd. Arnold, t. II, v. 9747-60, v. 10283-86 et v. 13266-70. Voir M. Delbouille, «Le témoignage de Wace sur la légende arthurienne », Romania, 74, 1953, p. 185-92.

${ }^{2}$ Dans Erec et Enide, la première occurrence est une simple mention associant implicitement l'appartenance à la Table Ronde et la renommée ( (De la Table Reonde estoit, /An la cort molt grant los avoit», éd. M. Roques, Paris, Champion, 1981, v. 83-4); une deuxième allusion accompagne l'énumération de trente et un chevaliers lors de la venue d'Erec à la cour (v. 1667ss) ; dans Le Conte du Graal, Ygerne demande à Gauvain s'il fait partie de la Table Ronde (éd. F. Lecoy, Paris, Champion, 1984, v. 7872-73).

${ }^{3}$ Par exemple, «The Round Table», Arthurian Tradition and Chretien de Troyes, New York, Columbia University Press, 1949, p. 61-8 ; J. Marx, La légende arthurienne et le Graal, Paris, PUF, 1952.

${ }^{4}$ Voir A. Micha, "L'origine de la Table du Graal et de la Table Ronde chez Robert de Boron ", Romance Philology, 9, 1955-56, p. 173-76. Sur la Cène comme modèle: L. H. Loomis, "The Table of the Last Supper in Religious and Secular Iconography », Art Studies, V, 1927, p. 71-88 et A. Barb, «Mensa sacra. The Round Table and the Holy Grail», Journal of the Warburg and Courtauld Institutes, 19, 1956, p. 40-67.

${ }^{5}$ Pensons aux travaux sur la table de Winchester et sur la récupération du mythe littéraire : voir par exemple King Arthur's Round Table : an Archeological Investigation, dir. M. Biddle et alii, Woodbridge, Boydell, 2000; A. Chauou, L'idéologie Plantagenêt. Royauté arthurienne et monarchie politique (XII ${ }^{e}$ XIII ${ }^{e}$ siècles), Rennes, Presses Universitaires de Rennes, 2001, p. 138-44 et C. Daniel, Arthurianisme et littérature politique, thèse soutenue sous la direction de J. CL. Thioler, Paris XII, 2002, p. 871ss et p. 908ss.

${ }^{6}$ Comme en témoigne par exemple sa reprise dans Perceforest : voir A. Berthelot, «Table Ronde et Franc Palais », Die Ritterorden im Mittelalter, éd. D. Buschinger, Wodan, 67, Greifswald, Reineke Verlag, 1996, p. 1-9 et G. Roussineau, «Ethique chevaleresque et pouvoir royal dans le Roman de Perceforest », Actes du 14e congrès international arthurien, Rennes, Presses Universitaires de Rennes, 1985, t. II, p. 521-35.
}

Cahiers de Recherches Médiévales, 14, 2007 
l'ambition encyclopédique du roman postérieur à Chrétien de Troyes'. La Table Ronde n'est pas qu'un mythe politique : elle a aussi une dimension poétique.

Les Merveilles de Rigomer, roman en vers composé vers $1250^{8}$ par un certain Jehan, est un de ces textes, souvent sous-estimés, où l'héritage de Chrétien de Troyes conduit, selon les jugements, soit à un renouvellement du genre romanesque, soit à sa décadence. L'intrigue en est complexe 9 . On peut néanmoins dégager trois parties $^{10}$. Dans la première (v. 1-6402), Lancelot triomphe d'épreuves aventureuses jusqu'à son arrivée à Rigomer où il est ensorcelé par une lance et un anneau magiques qui le transforment en cuisinier. Dans la deuxième (v. 6403-14776), Gauvain conduit une quête de cinquante-huit chevaliers pour le délivrer. Dans la troisième (v. 14777-15916), Gauvain n'épouse pas la dame de Rigomer mais lui promet un époux qu'il va chercher à la cour d'Arthur. Un fragment relance les aventures : Arthur repart avec Lancelot (v. 15917-17271) $)^{11}$. Le récit reste inachevé.

Dans ce récit d'aventures entrelacées, la Table Ronde, qui sert de modèle aux listes de chevaliers aventureux pourrait jouer un rôle fondamental, car c'est elle qui lance les preux sur les chemins de l'aventure et qui organise par ce biais le récit. Cependant dans ce roman sans Graal, la haute table spirituelle est remplacée par la nourriture, leitmotiv qui signale le potentiel parodique du texte, en relation avec un renouvellement et de la société médiévale et de l'écriture romanesque, potentiel dont témoignerait aussi un jeu sur l'expression «maisnie Arthur ", comme désignation à la fois d'une élite chevaleresque et d'un avatar de la «maisnie Hellequin».

\footnotetext{
${ }^{7}$ Sur la Table Ronde comme image du monde, voir H. Eberlin-Westhues, «König Arthurs Table Ronde. Studien zur Geschichte eines literarischen Heerschaftszeichens », Der altfranzösische Prosaroman, Actes du colloque de Würzburg, 1977, dir. E. Ruhe et R. Schwaderer, Munich, Fink, 1979, p. 184-269. Sur la rime monde/ ronde, voir É. Baumgartner, «Jeux de rimes et roman arthurien», Romania, 103, 1982, p. 550-60, repris dans De l'histoire de Troie au livre du Graal, Orléans, Paradigme, 1994, p. 49-59. Ajoutons au relevé d'É. Baumgartner que l'on trouve cette rime dans le Roman de Hem de Sarrazin, daté de 1278 (éd. A. Henry, Paris, Les Belles Lettres, 1939) aux vers 335-36. Sur la rotondité de la Table et les romans en prose, voir É. Baumgartner, «La couronne et le cercle : Arthur et la Table Ronde dans les manuscrits du Lancelot-Graal», Texte et Image. Actes du colloque de Chantilly, Paris, Les Belles Lettres, 1982, p. 191-200, repris dans De l'histoire de Troie au livre du Graal, p. 361-77.

${ }^{8}$ Pour Th. Vesce (The Mervels of Rigomer, ed. and trans., New York, Londres, Garland, 1988, p. xv) le roman daterait de la fin du XII siècle. Je pencherais plutôt pour la proposition de B. Schmolke-Hasselman (Der arthurische Versroman von Chrétien bis Froissart, Tübingen, Niemeyer, 1980, p. 16), qui le date des années 1250-68. Voir aussi, plus loin, la note 34.

${ }^{9}$ Pour un résumé, voir A. Micha, "Miscellaneous French Romances in Verse », Arthurian Literature in the Middle Ages, éd. R. S. Loomis, Oxford, Oxford University Press, 1959, p. 386 et P. Noble "The Role of Lorie in Les Merveilles de Rigomer», Bulletin Bibliographique de la Société Internationale Arthurienne, 48, 1996, p. 283.

${ }^{10}$ Mes références renvoient à l'édition W. Foerster et H. Breuer, Dresden, 1908-1915, 2 vol.

${ }^{11}$ Voir F. Carapeza, «Le fragment de Turin », Romania, 119, 2001, p. 76-112.
} 


\section{«Dix petits nègres »: quand le conte compte}

Le nombre de chevaliers invités à la Table Ronde a varié. Ils sont douze (ou treize en comptant le Siège Périlleux) lorsque prévaut le rapprochement avec la table de la Cène, d'abord dans le Joseph de Robert de Boron, puis dans Perlesvaus et le Lancelot en prose. Cependant Wace ne précisant pas le nombre d'élus, la tendance a été à l'inflation: la Table Ronde a eu 50 ou 51 places $^{12}$, voire 150 (dans la Suite du Merlin $d u$ cycle Post Vulgate) ou $300^{13}$, cette plasticité lui permettant d'intégrer de nouveaux chevaliers au fur et à mesure que le roman arthurien se renouvelait et élargissait son personnel ${ }^{14}$. Cependant à cette euphorie expansionniste répond la sombre tristesse d'une Table Ronde qui perd ses valeureux, comme dans La Mort le Roi Artu ${ }^{15}$. Le début de la Queste du Saint Graal montre une Table Ronde se vidant dans la dépression générale ${ }^{16}$. Les évocations de la Table donne donc lieu soit à des dénombrements globaux, soit à des listes, échos lointains des énumérations épiques, comme dans Floriant et Florete (v. 945ss) ou Les Premiers faits du roi Artu (p. 91213) : en elle se rejoignent le nom et le nombre.

Les références à la Table Ronde rythment Les Merveilles de Rigomer: dans la première partie (les aventures de Lancelot), la Table semble de peu de poids ; en revanche la quête menée par Gauvain est structurée par une liste de chevaliers qui renvoie, de façon décalée, au modèle de la Table Ronde; les aventures de Gauvain et Arthur qui prennent la suite semblent confirmer la mise au rebut de cette grandiose construction.

Dans l'ouverture traditionnelle de la première partie, lorsqu'une demoiselle vient chercher de l'aide (v. 79ss), on attend en vain une mention de la Table Ronde. Plus loin, Lancelot, alors même qu'il s'est à peine éloigné de la cour, n'est plus reconnu de tous, comme si la Table Ronde ne jouait pas son rôle, même dans un environnement proche (v. 1881). La première référence à cette auguste institution est faite par Lancelot, qui se présente à des voleurs (v. 763-64), ce qui est dévalorisant; de même, plus loin, c'est un chevalier vaincu qui dit avoir entendu parler «De la loial Table Reonde / En cui toute prouece habonde» (v. 3267-68). Ce n'est que très tardivement, à proximité de Rigomer, que le roi Frion, qui a été à la cour d'Arthur quatorze ans plus tôt et qui connaît le «los» de Lancelot (v. 4484), demande des nouvelles de la cour, de Gauvain et d'Artus, et mentionne Keu, Wales li Caus, le Chevalier au Cor, le Valet au cercle d'or, Yonet, Lanval, Waheriet, Sagremor, Engrevain, ainsi que Carahés, le fils du roi Lot (v.4486ss). Cette énumération mentionne onze chevaliers : si l'on ajoute Lancelot, la « liste Frion» comporte douze

\footnotetext{
${ }^{12}$ Dans le Merlin en prose, cinquante chevaliers y prennent place, le Siège Périlleux restant vide (éd. A. Micha, Genève, Droz, 1979, §49).

${ }^{13}$ Les Premiers faits du roi Arthur, version Vulgate de la suite Merlin, éd. dans Le Livre du Graal, sous la dir. de Ph. Walter, Paris, Gallimard, La Pléiade, 2001, p. 1324.

${ }^{14}$ C'est ainsi par exemple que Floriant, à la fin de Floriant et Florete, devient chevalier de la Table Ronde (éd. A. Combes et R. Trachsler, Paris, Champion Classiques, 2003, v. 2134-36). ${ }^{15}$ Éd. J. Frappier, Genève, Droz, 1954. Dans un premier temps, 72 chevaliers sont remplacés (p. 138) sans que leur identité ni celle des remplaçants ne soient précisées (p. 138), ce qui est un signe de l'immense fatigue du monde arthurien ; puis après les combats de la fin du roman, un décompte sinistre rythme la chute du royaume : ne restent que 72 chevaliers, puis 4, puis 2 . ${ }^{16}$ Éd. A. Pauphilet, Paris, Champion, 1980.
} 
chevaliers, comme la Table Ronde inspirée de la Cène. L'expression table reonde n'est pas employée, mais ce discours de Frion est appelé par le fait que Lancelot s'est présenté comme appartenant à la «maisnie del roi » (v. 4481). Cette énumération a six noms en commun avec celle d'Erec et Enide (v. 1671ss): manquent néanmoins Erec, Gornemant et Melian, qui sont des figures importantes dans l'œuvre du champenois, comme si le séjour de Frion était antérieur aux temps évoqués par Chrétien. La première partie s'achève sur l'échec de Lancelot, finalement prisonnier à Rigomer : il appartient à la Table Ronde, mais cela n'a guère servi.

Le roman est relancé par une intervention de Jehan (v. 6429-44) et s'ouvre sur une nouvelle scène de cour, à Carduel, traditionnelle, où le roi attend une aventure pour se mettre à table. Paraît alors Maudin, envoyé à la cour par Lancelot qui l'a vaincu précédemment: il est très bien reçu et intégré à la Table Ronde (v. 6552). N'y a-t-il pas inversion du motif attendu ? N'est-ce pas curieux d'admettre un chevalier dans un épisode d'introduction (et non dans un dénouement), et qui plus est, un vaincu, dont on ne reparlera plus, et dont le nom n'est d'ailleurs pas repris au moment de son intronisation, alors même que normalement la gloire de la Table Ronde est fermement associée au nom, à l'identité, à la renommée? L'institution chevaleresque semble avoir des ratées. D'ailleurs, même si un certain nombre de chevaliers sont prêts à se mettre en aventure, Gauvain les retient : ce n'est qu'un faux départ (v. 6589ss). Quand la décision de partir est finalement prise, c'est la Toussaint, il faut laisser passer l'hiver (v. 7020). Après une reverdie, une nouvelle cour se réunit. Gauvain appelle ses compagnons à se mettre en quête de Lancelot. Suit une énumération, la liste de Gauvain, qui fait écho à la liste de Frion de la première partie (v. 7061-100), et se compose de cinquante et un chevaliers. Cette énumération reprend les noms de tous les chevaliers de la liste Frion, excepté Lancelot, ce qui est logique puisqu'il est prisonnier, et Wales li Caus, ce qui est plus étrange, car ce chevalier est l'un des piliers de ce type d'énumération ${ }^{17}$. Sont intégrés des personnages de Chrétien (l'Orgueilleux de la Lande, Gornemant, Melian) et des continuations, comme Bran de Lis. Certains chevaliers de la liste d'Erec sont reconduits comme le Laid Hardi ou le Valet de Quinquareus (Quitareus dans Erec v. 1693). La liste témoigne du plaisir du jeu verbal. Les désignations périphrastiques sont rapprochées: le Chevalier au Cor et le Valet au Cercle d'Or appellent le Chevalier à la Cotte Maltaillée (v. 7074-76) ${ }^{18}$. L'auteur varie le rythme : un, deux ou trois noms saturent chaque vers. Le jeu sur les dédoublements (déjà lancé par Chrétien qui mentionne deux Yvain) est développé : il y a cinq Yvain, répartis en deux mentions. Quand ce n'est pas le son (Greminés résonne dans Gremion v. 707172, Brandelis v. 7070 s'inverse presque en Elibran v. 7093), c'est un rapprochement sémantique qui assure la cohérence : est-ce un hasard si Marmans du Cop vient juste

\footnotetext{
${ }^{17}$ Erec et Enide, v. 1696. Il figure parmi les quinze plus grands barons d'Arthur dans Floriant et Florete (v. 945ss).

${ }^{18}$ Dans mon édition de la fin du Tristan en prose donnée par le manuscrit BnF fr. 757 (à paraître chez Champion), j'ai émis l'hypothèse d'un rapport entre le Valet à la Cotte Maltaillée et le Chevalier au Cor, rapport que pourrait confirmer cette énumération qui les rapproche.
} 
avant Barnaains (v. 7088), qui rappelle le Balaain de l'aventure du Coup Périlleux ${ }^{19}$ ? Nous aurions donc là une liste qui reprendrait le topos des cinquante membres associés à la Table Ronde sur les conseils de Merlin chez Robert de Boron (\$49). Quelques indices suggèrent cependant un décalage : le compte n'est pas juste (on arrive à 52 chevaliers en comptant Gauvain); la Table Ronde n'est pas mentionnée explicitement; c'est Gauvain, et non le roi ou Merlin, qui choisit. Par ailleurs, comment croire que ce groupe constitue une élite quand les deux derniers nommés sont Carahués Briébras, un estropié, «Et Jaidons qi sovent fu las/ En la voie d'armes porter/ Ains qu'il venist a Rigomer» (v. 7098-100) ? Le plus étonnant est que, dans un second temps, Gauvain accepte tous ceux qui souhaitent faire partie du groupe et la troupe s'élève rapidement à plus de cinq cents hommes (v.7111). Même si, comme pour la vraie Table Ronde, on prête serment, la négociation qui suit démythifie la grandeur de l'institution merlinesque : le roi, désolé de voir sa cour désertée (v.7121), obtient une diminution du nombre des chevaliers engagés; Gauvain concède que seuls quarante l'accompagneront, et le roi finit par en accorder soixante (v. 7135-36). Finalement, alors même que le nombre semblait un enjeu de taille, on ne saura pas exactement à ce moment-là combien sont les preux, et on restera dans l'ignorance de leur identité. Ce n'est qu'une fois les chevaliers en route qu'on apprend qu'ils sont «sisante chevaliers .II. mains» (v. 7163): curieuse expression, qui sent le marchandage et qui signale un décalage, ce nombre étant inédit dans la tradition de la Table Ronde et ne semblant guère porteur de symbolisme. Le roman évolue donc à partir d'une Table Ronde réduite à treize, quasi légendaire dans la bouche de Frion, que rien ne vient réactualiser.

La deuxième partie du récit est structurée comme le roman d'Agatha Christie, Dix Petits Nègres. Tout se joue sur le mode du décompte. La troupe de Gauvain passera successivement à 57 chevaliers, puis 56,55 , etc..., ce qui explique peut-être que dès le début les chevaliers sont «sisante .II. mains", soixante moins deux : le décompte a commencé avant même le début des aventures. À la Table Ronde heureuse des temps de fondation, où l'on élisait les meilleurs, a succédé un groupe qui s'émiette inexorablement. Gauvain est fait prisonnier le premier : «Lors s'en vont li .1. .vij.» (v. 7469). Puis c'est au tour de Sagremor (v. 7625ss). Disparaîtront ensuite six autres chevaliers, successivement Engrevain (v. 7994), Blioblieris (v. 8449), Yvain del Leoneil (v. 8573), Gaudins li Bruns de la Montagne (v. 8864), Clices de Gresse (v. 9113), et Waheriés (v. 9495). Chacun connaîtra des aventures, dont la plupart évoquent, plus ou moins, les romans de Chrétien ou de ses successeurs. Le décompte s'accompagne d'un effet anthologique. Ce seront cinquante chevaliers qui arriveront à la Lande de Rigomer (v. $9834^{20}$ ), ce qui pourrait correspondre au nombre de places de la Table dans le Merlin de Robert de Boron.

La deuxième partie se présente donc comme un réajustement sous l'effet conjugué des négociations entre Gauvain et Arthur et de l'aventure. Le modèle affiché d'Agatha Christie est une nursery rhyme anglaise, sur une trame qui, encore aujourd'hui, dans de nombreux récits pour enfants, permet l'apprentissage du dénombrement. Ne peut-on imaginer qu'au XIII ${ }^{\mathrm{e}}$ siècle existaient des «comptines »,

19 On peut supposer que Les Merveilles de Rigomer sont postérieures au Huth Merlin (v. 1235-40).

${ }^{20}$ Il faut lire «Qui dusques a .L. i sunt. » 
pour compter, de ce type, dont l'auteur se serait inspiré? Ce ne serait guère surprenant, étant donné la fréquence des emprunts de type folklorique dans ce texte $^{21}$. L'entrelacement est ici revu, déplacé et recomposé sur ce modèle ${ }^{22}$.

La suite du récit propose d'autres listes de chevaliers, qui reprennent en partie des éléments de la liste de Frion ou de la liste de Gauvain : à chaque fois on note une inflation numérique et un échec à redonner sens à la Table Ronde. Ainsi la première énumération lors des joutes des landes de Rigomer contre les Irlandais est l'occasion d'une sélection comprenant onze chevaliers qui figurent tous dans l'une des deux listes fondatrices (sauf Tristan, qui néanmoins se trouve dans la liste d'Erec et Enide v. 1687) ${ }^{23}$, sans que la Table Ronde soit explicitement mentionnée. Un groupe plus nombreux, composé des cinquante chevaliers arrivés à Rigomer, «la maisnie au roi Artu» (v. 9835), est évoqué, et enfin plus largement la troupe de sept cents preux constituée après des ralliements massifs (v. 10162).

Le deuxième jour, sept Bretons sont nommés, qui appartiennent soit à la liste de Gauvain, soit à celle de Frion (v. 10479ss). À ces noms familiers s'opposent ceux des Irlandais, inédits et souvent pittoresques: Boutincostiaus, Ginemans, Taibruns (v. 10487ss). Une autre énumération de Bretons mentionne alors surtout des noms qui ne figurent ni dans les listes, ni dans la vulgate de la tradition arthurienne (le roi de Mon Marouac, v. 10525; Midolias de Galöee, v. 10539), comme si l'exotisme des Irlandais était contagieux. Ces onze noms sont le doublon estrange de la liste donnée à l'occasion du premier jour de tournoi, qui comporte elle aussi onze membres. Pourtant la dernière énumération de Bretons (v. 13545ss) comporte à nouveau des noms familiers, figurant tous dans la liste de Gauvain (Bedios le connétable v. 13624 est le Bedinous du vers 7096). À ces treize noms familiers s'opposent ceux des combattants irlandais, présentés non individuellement, mais par groupes, comme autant de peuples échappés des encyclopédies: Chenelius (v. 13669ss), Pismëi (les Pygmées), Cocus, Torvains et autres Popelicans (v. 13677ss). Ce retour à des noms traditionnels et à un nombre qui correspond à la Table des origines prépare la victoire bretonne et laisse supposer un possible retour de la Table Ronde, jamais accompli cependant dans le reste de l'œuvre.

\footnotetext{
${ }^{21}$ Voir Marie-Luce Chênerie, «Un recueil arthurien de contes populaires au XIII ${ }^{\mathrm{e}}$ siècle ? Les Merveilles de Rigomer», Réception et identification du conte depuis le Moyen Âge, textes réunis par Michel Zink et Xavier Ravier, Université de Toulouse le Mirail, 1987, p. 39-49 et M. Lecco, "Mais l'aventure revient ja. Retaggio folclorico e scrittura nelle Merveilles de Rigomer», Saggi sul romanzo del XIII secolo, Alessandria, Dell'Orso, 2003, p. 55-69.

${ }^{22}$ Sur la structure des Merveilles de Rigomer, voir D. Kelly, «Multiple Quests in French Verse Romance : Merveilles de Rigomer et Claris et Laris», L'Esprit créateur, 9, 1969, p. 257-66; N. Lacy, «Les Merveilles de Rigomer and the Esthetics of Post-Chrétien Romance », Arthurian Yearbook, 3, 1993, p. 75-88.

${ }^{23}$ Que penser de cette «apparition»? D'une part il faut être prudent, car les listes de noms sont souvent perverties dans les copies et ce n'est pas parce que Tristan ne figure pas dans la liste de Gauvain dans le texte sur lequel je travaille qu'il ne figurait pas dans la version originale. D'autre part, on peut penser que cette mention renvoie à l'idée d'une ouverture de la Table Ronde, mouvante, sans cesse renouvelée, moins stricte car un peu décadente et surtout inopérante.
} 
En effet, la Table Ronde n'est plus une institution vivante dans la suite des Merveilles de Rigomer : quand Gauvain libère les prisonniers de Rigomer, sont bien mentionnés les sept preux, objets du décompte de la deuxième partie (Gauvain est ailleurs, chez la fée Lorie), mais en tout ce sont 140 chevaliers, qui resteront pour la plupart anonymes, qui sont délivrés (v. 14299). La chevalerie a perdu son épine dorsale; elle est un groupe aux contours flous, jouant sur les grands nombres plus que sur l'élite d'une Table à douze voire cinquante compagnons choisis.

La troisième partie, après la délivrance de Rigomer, s'ouvre sur une cour printanière et une demoiselle qui vient chercher de l'aide (v. 14827ss) : l'aventure cependant piétine, de faux départ en faux départ, et finalement, la Table Ronde ne perce pas: Miraudiaus tombe malade de peur sous l'effet de la réputation de Gauvain (ce qui signale bien à quel point la Table Ronde, fondée sur la renommée, peut bloquer le monde chevaleresque). À la fin de cette aventure, qui termine le manuscrit de Chantilly, Miraudiaus «[...] fu de la Table Reonde / Ou toute la prouece abonde» (v. 15915-16). C'est sur cette intégration à la Table Ronde que se termine cette version. C'est un vaincu que ce nouveau chevalier: les deux seuls nouveaux chevaliers de la Table Ronde explicitement nommés dans Les Merveilles de Rigomer sont deux vaincus, Maudin et Miraudiaus. La Table Ronde institutionnelle n'est plus qu'un lot de consolation, que des substituts plus modernes, comme la liste de Gauvain, ne parviennent pas à revigorer.

Dans la suite qui figurait dans le manuscrit de Turin (qui est peut-être d'un autre auteur), le récit s'ouvre à nouveau sur une cour d'Arthur où une demoiselle vient chercher de l'aide: personne, excepté Arthur, ne répond d'abord à son appel (v. 15590ss), et plus de cinq cents chevaliers, en larmes de voir le roi partir, décident finalement de suivre celui-ci (v. 16092). Cette maisnie Arthur, trop nombreuse, se réduit finalement à cinquante trois chevaliers (v. 16123). Comme dans la liste de Gauvain, s'opposent un groupe très large de cinq cents hommes et une troupe d'élite, d'un peu plus de cinquante preux. La liste d'Arthur ne coïncide cependant pas exactement avec celle de Gauvain : sur les vingt-deux noms donnés, seuls seize sont communs. Finalement le roi partira seul avec Lancelot et cette maisnie restera sans postérité, le roman étant inachevé.

Ainsi la Table Ronde institutionnelle ne fonctionne pas bien dans Les Merveilles de Rigomer. Ses avatars et ses renouvellements sont décevants. Parce que la Table Ronde avait un fondement spirituel, qui est ici mis à l'écart, au profit des nourritures terrestres. Parce que la Table Ronde, dans ce roman marqué par le folklore, est relue par rapport à la maisnie Arthur du folklore, appelée aussi maisnie Hellequin.

\section{À table!}

La première table que mentionne le roman se trouve dans la Ruiste Valee (v. 452) et elle est évoquée dans un épisode qui parodie la scène du Graal : Lancelot mange d'abord dans le silence le plus complet, puis on ne lui répond pas quand il parle (v. 475); la nourriture est abondante (v.477); le héros repart seul au petit matin. Mais la table n'a rien de sacré : à la fin du repas, tous s'assoient dessus et l'on découvrira que ce n'est là qu'une assemblée de voleurs (v. 481ss). Quand au terme de cette aventure Lancelot se présente comme appartenant à la Table Ronde (et, fait notable, c'est la première occurrence du motif dans le roman: v. 762-63), on a 
l'impression que le détournement de la Table du Graal appelle le souvenir de la Table Ronde : la rime traditionnelle «reonde / monde» (v. 763-64) n'est cependant pas associée à l'ambition grandiose de la Table Ronde, mais à l'évocation de biens matériels («l'avoir del monde») et la mention de l'auguste institution ne semble susciter aucune réaction particulière chez le vicomte.

Par ailleurs, la nourriture est omniprésente dans le roman. Repas, aventure et plaisir du conte sont explicitement associés : "Car qant on a but et mangié, / Les gens sunt plus enromancié» (v. 6487-88). La nourriture est une obsession: «Se Gaudin consuist de plain, / Il ne manjast jamais de pain» (v. 8981-82) ; «Ainc nus om ne manja de dens, / Qui vëist millor par senblant» (v. 9132-33, v. 1867ss) ${ }^{24}$. L'auteur n'hésite pas à nous donner des menus, des listes de denrées (v. $815 \mathrm{ss}$, v. $1867 \mathrm{ss}$, v. $2137 \mathrm{ss}$, v. $2337 \mathrm{ss}$ ) ; les héros ont souvent faim (quand Lancelot croise la chasse nocturne, cela n'éveille en lui que l'idée de «lardés» et de «braons» v. 1230; Gauvain prisonnier de Gaudionés a grand faim v.7429-ss; quand Sagremor court le cerf, il ne rêve pas du Graal, mais d'une "haste de cerf lardee» v. 7686).

Par ailleurs on ne reviendra pas sur le potentiel parodique des scènes de cuisine à Rigomer ${ }^{25}$. Les Merveilles de Rigomer parodieraient la quête du Graal en la privant de son enjeu merveilleux ou sacréé ${ }^{6}$. Quand Jehan présente son récit comme un «romans [...] biaus et plaisans, et le plus lais» (v. 6434), ne fait-il pas référence à un récit «laïc», sans Graal? On ne pourra pas s'étendre ici sur la dimension parodique, soutenue par la présence de larrons, de vilains, par des combats avec des armes burlesques (v. 3397ss), des caricatures (v.3470ss), le tout associé à une discrète (?) critique de la chevalerie (v. 3519ss) ${ }^{27}$.

Finalement la Table, prise au pied de la lettre, privée de Graal, est à l'image d'une société en pleine évolution et d'un roman en pleine mutation. Comme ce

\footnotetext{
${ }^{24}$ Cette obsession langagière donne lieu à des expressions inhabituelles et pittoresques : aux v. 1714-18, la ramposne de Lancelot à un vaincu à qui il a coupé le pied, repose sur une allusion à un soc, c'est-à-dire un ragoût (voir M. Dubos, «Notules lexicologiques ", Romania, 78, 1957, p. 392).

${ }^{25} \mathrm{R}$. Trachsler, «Lancelot aux fourneaux : des éléments de parodie dans les Merveilles de Rigomer?», Vox Romanica, 52, 1993, p. 180-93 ; P. Ihring, "Wunder zum Lachen : Die Komische Entzauberung des arthurischen merveilleux in zwei altfranzösischen Versromanen aus dem 13. Jahrhundert, Meraugis de Portlesguez et Les Merveilles de Rigomer", Das Wunderbare in der arthurischen Literatur, éd. F. Wolfzettel, Tübingen, Niemayer, 2003, p. 175-91 ; S. Gordon, "Culinary Comedy in French Arthurian Romance», Medievalia et Humanistica, 30, 2003, p. 15-31.

${ }^{26}$ D'où la coprésence de deux discours parallèles, dont la hiérarchie est problématique. On peut en effet lire Les Merveilles de Rigomer comme une allégorie morale, les sept chevaliers retenus et délivrés représentant les péchés capitaux, par exemple.

${ }^{27}$ Voir L. Walters, «Chantilly Ms 472 as a Cyclic Work?», Cyclification : The Development of Narrative Cycles in the Chansons de geste and the Arthurian Romances, ed. B. Besamusca, W. P. Gerritsen, C. Hogetoorn et O. Lie, Amsterdam, Royal Academy of Arts and Sciences, 1992, p. 135-39; L. Walters, "The Formation of a Gauvain Cycle in Chantilly Manuscript $472 »$, Neophilologus, 78, 1994, p. 29-43 et «Parody and Moral Allegory in Chantilly Ms $472 »$, Modern Language Notes, 113:4, 1998, p. 937-50.
} 
vilain (v. 3003ss), preux et courageux, hospitalier, vêtu d'un manteau d'hermine, époux d'une très sage femme et entouré d'une maisnie de chevaliers et de sergents, qui signale le renouvellement social qui accompagne ce milieu du $\mathrm{XIII}^{\mathrm{e}}$ siècle, le roman arthurien, noble, emprunte non seulement à l'historiographie et à des sources d'élite, mais aussi, désormais ostensiblement, au folklore. Même si le soir, chez le vilain on raconte des fabliaus (v.3060) alors que chez le chevalier mahaigniés on raconte des récits au sujet «Des rois et des dus et des contes, / Des aventures et des lais» (v. 2732-33), il est clair que la richesse, la santé et une certaine courtoisie vigoureuse sont du côté du vilain alors que le chevalier est "mahaigniés ». D'où peut-être le projet de redonner vigueur au roman, en mêlant les genres, comme se mêlent les ordres sociaux. Le roman arthurien traditionnel, avec sa Table Ronde et son Graal, ne sont plus opératoires : le nouveau roman prend le relais ${ }^{28}$.

\section{La maisnie Artus}

Le goût de l'auteur des Merveilles de Rigomer pour les motifs folkloriques me conduit à proposer une hypothèse au sujet de l'expression «maisnie Artu». Ces mots désignent souvent les chevaliers de la Table Ronde. Plutôt que de se dire de la Table Ronde, bien des personnages des Merveilles de Rigomer se présentent comme étant de «la maisnie del roi » (v. 4481) ou, plus fréquemment, de la «maisnie Artu». C'est le cas par exemple de Lancelot (v. 1754, v. 4944, v. 5825 , v. 8149) ou Sagremor (v. 7709). La plupart du temps, «Artu» rime avec «vertu» (v. 1754, v. 4944, v. 5825, v. $8149, \ldots)$ : cette association est beaucoup plus fréquente que la rime «ronde/ monde», traditionnellement associée à la Table Ronde, qui n'est employée ici que deux fois ${ }^{29}$.

Or, l'expression «maisnie Artu» désigne aussi fréquemment un avatar de la maisnie Hellequin. Le dominicain Étienne de Bourbon, dans la première moitié du $\mathrm{XIII}^{\mathrm{e}}$ siècle, raconte dans un exemplum qu'un paysan a vu au clair de lune une troupe somptueuse, qui se présente comme familia Arturi et qui ressemble à la mesnie Hellequin, ce qui se trouvait déjà vers 1210 dans les Otia imperialia de Gervais de Tilbury $^{30}$.

\footnotetext{
${ }^{28}$ La Table Ronde représente un certain ordre social (voir A. Guerreau-Jalabert, «Aliments symboliques et symbolique de la table dans les romans arthuriens (XII $-\mathrm{XIII}^{\mathrm{e}}$ siècles)», Annales ESC, 1992-93, p. 561-94 et D. Boutet, Charlemagne et Arthur ou le roi imaginaire, Paris, Champion, 1992, p. 150.

${ }^{29}$ Voir E. Baumgartner, «Jeux de rimes et roman arthurien », art. cit., p. 57.

${ }^{30}$ Voir Cl. Lecouteux, Chasses fantastiques et cohortes de la nuit au Moyen Âge, Paris, Imago, 1999, p. 74ss et Ph. Walter, éd., Le mythe de la Chasse Sauvage dans l'Europe médiévale, Paris, Champion, 1997. On trouvera le texte de Gervais de Tilbury dans Otia imperialia, éd. F. Liebrecht, Hanovre, 1856, p. 12ss et celui d'Étienne de Bourbon dans le Tractatus de diversis materiis (voir H. M. Flasdieck, Harlekin, Germanischer Mythos in romanischer Wandlung, Anglia, 61, 1937, p. 247-48 et C. Luttrell, «Folk Legend as Source for Arthurian Romance : the Wild Hunt», Essays in Memory of Lewis Thorpe, éd. K. Varty, Glasgow, 1981, p. 83-100). Sur la Mesnie Hellequin et ses avatars littéraires, on suivra avec le plus grand intérêt les travaux de K. Ueltschi, «Mythe et Littérature : le Moyen Âge de la Mesnie Hellequin dans la littérature moderne et contemporaine », communication au Colloque «Lectures du Moyen Âge », Lorient, du 31 mars au 2 avril 2005, à paraître ; et «Le Premier
} 
Par ailleurs, dans les Merveilles de Rigomer, le motif de la mesnie Hellequin est clairement présent, dans sa diversité ${ }^{31}$. Sans qu'il soit possible de développer ici, de nombreuses aventures peuvent être comprises comme mettant en scène des morts, des revenants : ainsi la chasse nocturne (v. 1195ss) ou l'apparition de nuit d'une troupe de dames et de chevaliers (v. $8271 \mathrm{ss}$ ), l'aventure de Cligès qui reprend le motif de l'Atre Périlleux (v.9103ss), le mort qui se réveille quand on lui ôte le tronçon qui traverse son cadavre (v. 8384ss). Si Sagremor joue un rôle important, en double de Gauvain (alors que les forces de Gauvain croissent à midi, celles de Sagremor baissent la nuit v. 7744), n'est-ce pas que son nom se termine par «mor» (qui évoque la mort)? Si les chevaliers bretons sont pris pour des diables d'enfer (comme Gauvain v. 11901ss), c'est peut-être que la maisnie Arthur est aussi une maisnie Hellequin. D'ailleurs parmi les troupes irlandaises qui combattent l'armée d'Arthur devant Rigomer figurent des «moine infernax» (v. 10278), qui pourraient bien être eux aussi des revenants. Qu'est-ce alors que Rigomer, sinon un au-delà, un bout du monde (mers, merc désigne la borne, la frontière), qu'il ne faut peut-être pas prendre au sérieux ${ }^{32}$ ?

On peut donc présenter l'hypothèse que l'expression «maisnie Artu» a été prise pour désigner dans Les Merveilles de Rigomer à la fois un groupe d'élite autour du roi, au même titre que la Table Ronde, et un troupe de revenants, comme la maisnie Hellequin. Parce que nous sommes à une époque où s'écrit, non la glorieuse jeunesse d'un royaume, comme chez Chrétien de Troyes, mais La Mort le Roi Artu, parce que la matière arthurienne semble s'épuiser à force de reprises, Arthur et ses hommes pourraient paraître morts. Mais pour l'auteur des Merveilles de Rigomer Arthur revient, au sens propre et au sens figuré du terme ${ }^{33}$ : et c'est ce que la dernière partie, qui fait d'Arthur le héros, a bien compris. À défaut d'Arthur, nous aurons toujours son fantôme.

Dans Les Merveilles de Rigomer, la Table Ronde ne remplit donc plus sa fonction. Quand elle est explicitement une table d'élite pour douze chevaliers, elle n'est que l'objet d'un discours tenu par un étranger nostalgique, Frion: elle ne semble plus jouer de rôle. Dans un deuxième temps, une nouvelle confrérie chevaleresque se recrée autour de Gauvain, mais ce n'est qu'une Table dénaturée : même si le nombre de chevaliers (autour de la cinquantaine) évoque une Table Ronde rénovée, l'élargissement est à la fois laxiste et arbitraire, et le compte n'est

Roi ou le Fils Méhaignié. À propos d'Arthur, d'Hellequin et des Plantagenêts », Colloque «Lignes et Lignages » organisé à Rennes les 13 et 14 octobre 2005, à paraître.

${ }^{31} \mathrm{M}$. Lecco, dans Il motivo della Mesnie Hellequin nella letterature medievale (Alessandria : Edizioni dell'Orso, 2001, p. 65-9), étudie les apparitions de la Maisnie dans Meriaduc et Les Merveilles de Rigomer. Voir aussi Ph. Ménard, "Une parole rituelle dans la chevauchée fantastique de la Mesnie Hellequin», Mélanges René Fromilhague, Littératures, 9-10, Université de Toulouse le Mirail, 1984, p. 1-11.

${ }^{32}$ Le nom de Rigomer annoncerait la veine parodique, d'autant qu'un saint Rigomer était bien vénéré au XIII ${ }^{\mathrm{e}}$ siècle et que donner son nom à un château féerique témoigne d'un décalage.

${ }^{33}$ Dans Le Chevalier au Pagegau, de la même façon Arthur remonte sur scène et se trouve confronté à des revenants. 
pas «rond». Finalement, on accueille à la vraie Table Ronde n'importe qui, les vaincus surtout. Sur un modèle vraisemblablement populaire, qui double et peut-être parodie l'entrelacement de la quête, la deuxième partie du roman élimine un à un les héros. L'absence du Graal, les nombreux indices parodiques qui dévalorisent socialement et spirituellement l'aventure, la primauté du matériel sur le spirituel, sont à rapprocher de l'omniprésence du thème de la nourriture et de la cuisine : la table est des plus terriennes et prosaïques, parce que le roman et le monde ont évolué. Finalement l'élite des chevaliers d'Arthur apparaît le plus souvent sous le nom de maisnie Arthur: le monde arthurien de Geoffroy de Monmouth et Chrétien est bien mort, mais il revient peut-être sous forme de "fantômes", de fictions, de romans: Les Merveilles de Rigomer seraient un avatar, sur le mode poétique, de l'espoir breton ${ }^{34}$.

Christine Ferlampin-Acher Université Rennes 2, CELAM/CETM

${ }^{34}$ Ces diverses approches me conduisent à privilégier pour la composition des Merveilles de Rigomer la date de 1250 plutôt que la fin du XII ${ }^{\mathrm{e}}$ siècle comme le propose Th. Vesce. 